\title{
One Minute After: Strong-Motion Map, Effective Epicenter, and Effective Magnitude
}

\author{
by Ta-liang Teng, Ludan Wu, Tzay-Chyn Shin, Yi-Ben Tsai, and William H. K. Lee
}

\begin{abstract}
This article reports the recent progress on real-time seismic monitoring in Taiwan, particularly the real-time strong-motion monitoring by the Taiwan Central Weather Bureau's telemetered seismic network (CWBSN), which is presently aiming at rapid reporting immediately after a large earthquake occurrence. If rapid reporting can be achieved before the arrival of the strong shaking, earthquake early warning will become possible. CWBSN has achieved the generation of the intensity map, epicenter, and magnitude within $1 \mathrm{~min}$ of the occurrence of a large earthquake. Both rapid reporting and early warning are principally applied to large $(M \gg 5)$ events; the requirement of on-scale waveform recording prompted CWBSN in 1995 to integrate strong-motion sensors (e.g., force-balance accelerometers) into its telemetered seismic monitoring system. Time-domain recursive processing is applied to the multi-channel incoming seismic signals by a group of networked personal computers to generate the intensity map. From the isoseismal contours, an effective epicenter is immediately identified that resides in the middle of the largest (usually the 100gal) contour curve of the intensity map. An effective magnitude is also defined that can be derived immediately from the surface area covered by the largest (usually the 100-gal) contour curve. For a large event with a finite rupture surface, the epicenter and magnitude so derived are more adequate estimates of the source location and of the strength of destruction. The effective epicenter gives the center of the damage area; it stands in contrast with the conventional epicenter location, which only gives the initial point of rupture nucleation. The effective magnitude reflects more closely the earthquake damage potential, instead of the classical magnitude definition that emphasizes the total energy release. The CWBSN has achieved in obtaining the above crucial source information well within $1 \mathrm{~min}$. This time can further be reduced to better than $30 \mathrm{sec}$, as illustrated by the example in this article, showing that earthquake early warning is indeed an achievable goal. The rapid reporting and early warning information is electronically transmitted to users to allow rapid response actions, with or without further human intervention.
\end{abstract}

\section{Introduction}

In real-time seismic monitoring, we are first aiming at rapid reporting immediately after the occurrence of a large earthquake. We hope that in less than a minute, information of epicenter, magnitude, and the intensity map will become available for emergency response purposes. If rapid reporting can be achieved before the arrival of strong shaking, earthquake early warning will become possible. Here, the possibility of earthquake early warning depends on the strong shaking ( $S$-wave) propagation delay time; therefore the epicentral distance between the source and the impact area matters. There is no early warning possible if the source is inside the impact area. However, in many situations, large metropolitan areas are not right over but are at some distance to large active faults (such as in California, Japan, and Taiwan). If, for example, the source area is $100 \mathrm{~km}$ from the impact area, the $S$-wave propagation time can be about 30 sec. For earthquake early warning, it is perhaps necessary to process the real-time data and determine the event parameters within $20 \mathrm{sec}$ in order to allow another $10 \mathrm{sec}$ for the activation of emergence response measures. Clearly, a larger epicentral distance with longer propagation delay time would allow for longer data processing time, but a larger epicentral distance would also cause stronger attenuation of the strong shaking, making early warning less important. A shorter epicentral distance with a shorter propagation delay time would put more severe limitation on the allowable data 
processing time, making the early warning problem more difficult, if not impossible. On the other hand, since both rapid reporting and early warning should only be applied to potentially damaging large events, a rapid determination of the magnitude is necessary. This calls for on-scale recordings that make it necessary that strong-motion sensors (e.g., force-balance accelerometers) be integrated into the telemetered seismic monitoring system. Taiwan has recently accomplished this integration on the CWBSN, and its progress on rapid reporting and early warning is reported here.

Of crucial importance to an effective rapid response to a damaging earthquake is an early determination of the epicenter, the magnitude, and particularly the intensity map. These data collectively constitute the essential elements in both rapid reporting and early warning operations. In the aftermath of a large earthquake, further escalation of property damage and loss of lives can be averted, or at least greatly reduced, if timely emergency service and rescue missions can be dispatched to the most needy areas. During past earthquake catastrophes such as the disastrous 1976 Tangshan earthquake, or even as recently as the 1994 Northridge earthquake and the 1995 Kobe earthquake, knowledge of shaking damage has sometimes been obtained by rather haphazard methods from air surveys to surveys conducted by dispatched field parties. In worst cases, damage reports often become available as late as days after the mainshock. Technological progress in remote sensing, signal processing, and data transmission has altered all this; we should be able to obtain this shaking intensity information right from the seismic network within minutes or even seconds.

A seismic computerized alert network was first discussed in concept by Heaton (1985). Besides the telemetry of on-scale waveforms, the crucial requirement that makes rapid reporting and early warning work is the rapid and accurate determination of the magnitude, especially the magnitudes for large $(M \gg 5)$ events. In Japan, a method of earthquake early warning has been employed by its bullet train operation. Nakamura (1989) reports an upgraded version of the Japanese UrEDAS system that estimates the earthquake magnitude from the predominant period of the initial $P$-wave motion. This method cannot always work since ruptures are usually finite and spatially heterogeneous and the beginning phases of large earthquakes are often similar to those of smaller earthquakes. A moment estimation method is proposed by Toksoz et al. (1990); this generally requires an assumption of the fault depth. A recursive stochastic deconvolution software to obtain real-time momenttensor magnitude from multi-channel input is demonstrated by Qu and Teng (1994) and Qu (1996). More software development is needed to reduce the computation time down to about $20 \mathrm{sec}$ in order for the method to be useful to rapid reporting and early warning.

Holden et al. (1989), in a report to the State of California, assessed the technical and economical feasibility of an earthquake warning system in California, and Bakun (1990) reported on an early warning alert system for the U.S. Geo- logical Survey. These findings are included in a National Research Council report (1991) recommending the implementation of real-time earthquake monitoring. Due to budget limitations, there has been no large-scale implementation in the United States. Some interesting progress is reported in a recent article on the Mexico City Seismic Alert System (Espinosa Aranda et al., 1995). It applies to a unique situation where the target area (Mexico City) is so far away from the source area that an $S$-wave propagation delay time of more than $70 \mathrm{sec}$ can be comfortably allowed for event identification, instead of on the order of 20 to $30 \mathrm{sec}$ for more ordinary cases. Nonetheless, the Mexico results represent an outstanding success in earthquake early warning. Of course, as the distance becomes large, it requires a much stronger (therefore much infrequent) source to make its impact felt in the target area.

The Central Weather Bureau's Seismic Network (CWBSN) in Taiwan has recently been upgraded to provide better information in a crisis. Automatic $P$ and $S$ pickings and rapid least-squares inversion routines have reduced the routine epicenter determination time in Taiwan from about 30 min to about $60 \mathrm{sec}(\mathrm{Wu}, 1996)$; this time can be further reduced by optimizing the processing hardware and software (Shin et al., 1996; Teng et al., 1996). However, the most crucial piece of information for emergency response purposes, that is, the shaking intensity, is generally not available until much later, depending on the nature of strong-motion networks. This shaking intensity information will not be supplied by an epicenter, which merely represents the initial point of rupture nucleation for a large event. But the realtime monitoring of the strong ground motions can provide the desired answer. With telemetered multi-channel strongmotion input, the CWBSN is able to generate within $1 \mathrm{~min}$ the intensity map, the epicenter, and the magnitude of an $M>5$ earthquake. This is accomplished by the CWBSN through (1) an upgrading of the telemetered seismic network to include both the weak- and strong-motion sensors, with a combined dynamic range of $166 \mathrm{~dB}$ from $10^{-8}$ to $2 \mathrm{~g}$, and (2) the use of efficient recursive seismic data processing software that parallel processes the incoming multi-channel signals by a group of networked PCs. The use of $2 g$ maximum digital accelerographs in the CWBSN ensures that the seismic signals are always on-scale. Data analysis systems have been implemented to rapidly generate the essential information for the rapid reporting and early warning objectives. In this article, a recent large event is used to illustrate the method that demonstrates the CWBSN's capability of obtaining the 1-min intensity map and the associated epicenter and magnitude estimates. This capability is found to be extremely useful in an area like Taiwan where the seismicity is high and damaging earthquakes occur often (Fig. 1). Software has been developed and installed on the CWBSN so that well within the 1-min time frame, the intensity map and epicenter-magnitude information can be electronically transmitted to the cognizant governmental agencies, the public utilities, and the communication and transportation systems, where 
desirable early emergency response operations can be triggered to mitigate further earthquake loss.

\section{CWBSN-Real-Time Seismic Monitoring in Taiwan}

Taiwan, with an area of $36,000 \mathrm{~km}^{2}$ (approximately $10 \%$ of California's area), is situated at the junction of the Ryukyu Island Arc and the Philippine Island arc. As the Philippine Sea plate subducts northward under the Eurasia plate along the Ryukyu trench, and the Eurasia plate subducts eastward under the Pacific plate off the southern tip of Taiwan, the entire Taiwan island is under a NW-SE compression with a measured convergence rate as large as $7 \mathrm{~cm} /$ yr. Large active thrusts and strike-slip faults are distributed virtually all over the island. The seismicity in Taiwan (Fig. 1) is about 3 to 5 times higher than that in California (Lee and Houck, 1977). A large part of the seismicity is found along the east coast and is offshore, while Taiwan's population centers are developed over the western plains where occasional large events constitute a large part of the earthquake damage history. In Taiwan, the operation of a telemetered seismic network began in the early 1970s, while onsite analog seismic recordings began as early as the turn of this century. In the late 1980 s, digital telemetry and digital recording were introduced to seismic monitoring operations, however, with only the conventional three-component shortperiod velocity sensors. There were then two telemetered seismic networks covering the entire Taiwan region: one being the CWBSN, operated by the Central Weather Bureau; the other being the TTSN (Taiwan Telemetered Seismic Network), operated by the Institute of Earth Sciences of the Academia Sinica. In the early 1990s, the TTSN stations were integrated into a new CWBSN of a total of 75 stations, all equipped with high-grade, matched three-component, shortperiod seismometers (Teledyne-Geotech S-13). Figure 2 shows the present CWBSN station map. Like any standard short-period seismic network, seismograms of the CWBSN are extensively clipped for any event larger than $M 4$. Thus these high-gain, short-period seismic networks provide little waveform information for large earthquakes, except perhaps the $P$-wave arrival times and the polarities.

In 1992, an important decision was made for CWBSN to add three-component high-quality, well-calibrated forcebalance accelerometers (FBAs) to all CWBSN stations (Shin, 1993; Teng et al., 1994). So a standard CWBSN telemetered seismic station consists of six components: three-component short-period velocity sensors and three-component acceleration sensors (FBAs). Also, an effort to develop and implement hardware and software for earthquake early warning was initiated (Lee, 1993; Lee et al., 1996). All signals of the CWBSN stations are digitally telemetered back to the Central Weather Bureau headquarters in Taipei for real-time recording and processing. The telemetry delay time is on the order of milliseconds and thus is omitted in this discussion. To date, this addition of FBAs has been implemented at 45 CWBSN stations (shown as solid triangles in Fig. 2). In con-

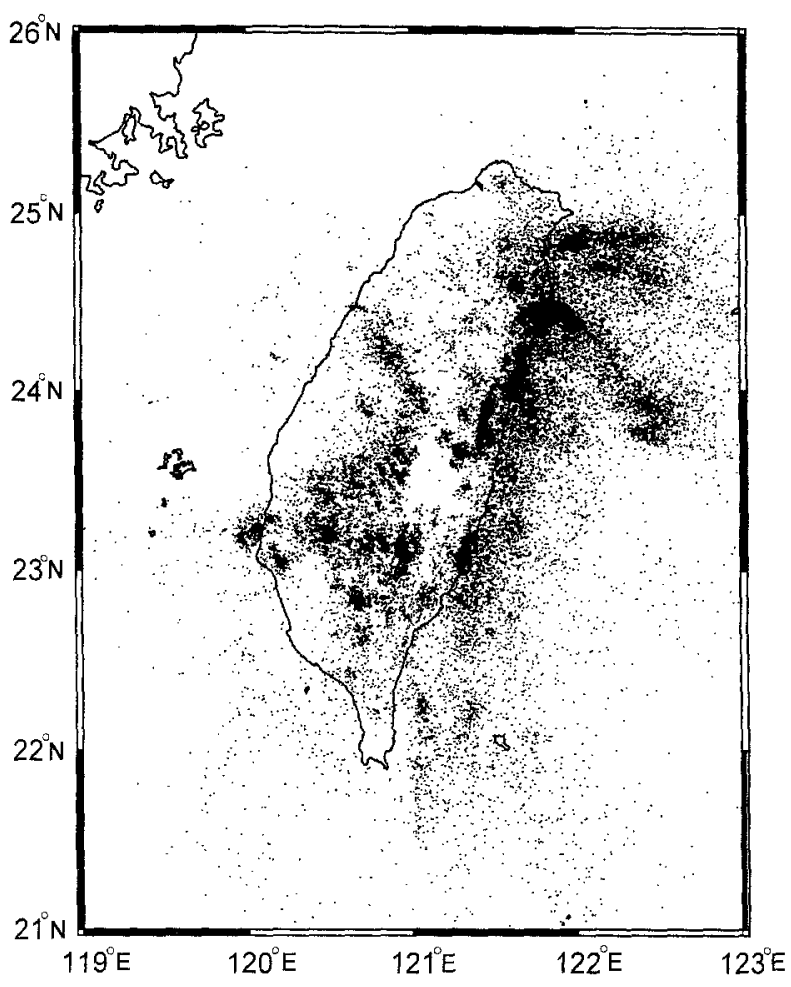

Figure 1. A seismicity map of Taiwan (from 1973 to 1996).

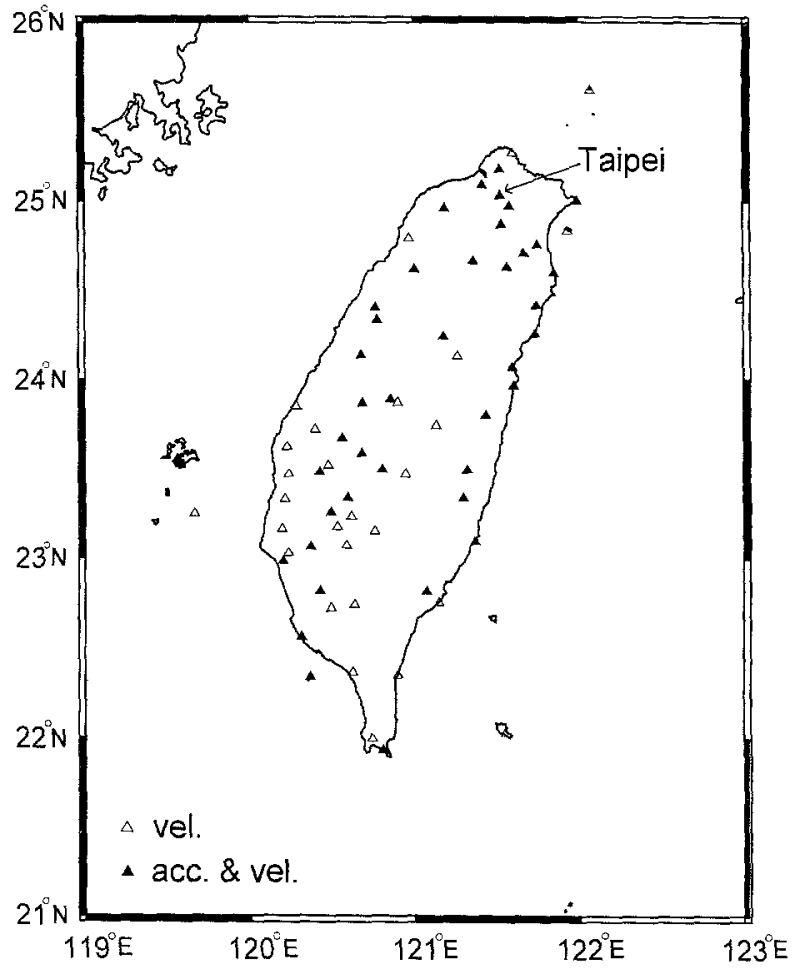

Figure 2. The CWBSN station map. 
tinuous digital data streams, strong-motion signals are telemetered back at 16-bit, 50 samples/sec, and velocity $(S-13)$ signals are telemetered back at 12-bit, 100 samples/sec, both in 1-sec data packets. Design and field tests have just been completed to upgrade all six-component data streams to be 16-bit and 100 samples/sec. This CWBSN upgrade will be completed in 1997. Figure 3 gives the system responses of the velocity sensors ( $S-13)$ and the FBA accelerometers (A900A). The system has a combined dynamic range of 166 $\mathrm{dB}$, or from $10^{-8}$ to $2 \mathrm{~g}$ in acceleration. Besides the upgraded CWBSN, the Central Weather Bureau in Taiwan has also installed 600 free-field digital strong-motion accelerographs with capability of absolute timing (Omega or GPS), and more than 40 real-time strong-motion monitoring arrays (each with 30 recording channels plus two timing channels) for buildings and bridges.

\section{Data and Method}

Figure 4 gives an example showing the quality of the CWBSN real-time FBA output for an $M 5$ event. Based on the global strong-motion database available so far, it is believed that the CWBSN will hardly ever clip its strong-motion waveforms. Figure 5 shows the typical integrated results of the FBA output that give the velocity and displacement traces for an $M 5.9$ event. In the near field of large events with high signal-to-noise ratio, the FBAs have been shown to be as effective a sensor as broadband seismometers such as the Streckeissen STS-1 (Wald et al., 1991). While the FBA is good for near-field recordings, it is not sensitive enough for recording teleseisms. Software has been developed to parallel process all incoming signals in the CWBSN (Fig. 6): a Vax system for processing the velocity $(S-13)$ signals for conventional seismic monitoring and a group of PCs networked into a fast parallel-processing system for real-time monitoring of both weak and strong motions. The latter is aiming at rapid reporting now; hopefully, it will approach early warning capability in the near future (Shin et al., 1996; Lee et al., 1996).

Both rapid reporting and early warning are only directed toward earthquakes large enough to cause damage, including those events large enough to inflict damage to areas well beyond the epicentral region. We shall refer to these large events as "consequential earthquakes," meaning that they are capable of inflicting significant damage on the societal well being. Though the smaller events are valuable for scientific research, they draw no attention from rapid reporting and early warning. In real terms, these consequential earthquakes refer to events of magnitude larger than perhaps $M$ 6 for rapid reporting and $M 7$ for early warning. Earthquake early warning will only work at distances 50 to $100 \mathrm{~km}$ away from the source region where there is enough propagation delay time to allow for time for data processing and response actions (Lee et al., 1996); therefore, early warning is especially directed toward really large earthquakes. An event much less than $M 7$ will indeed be considered as an "incon-

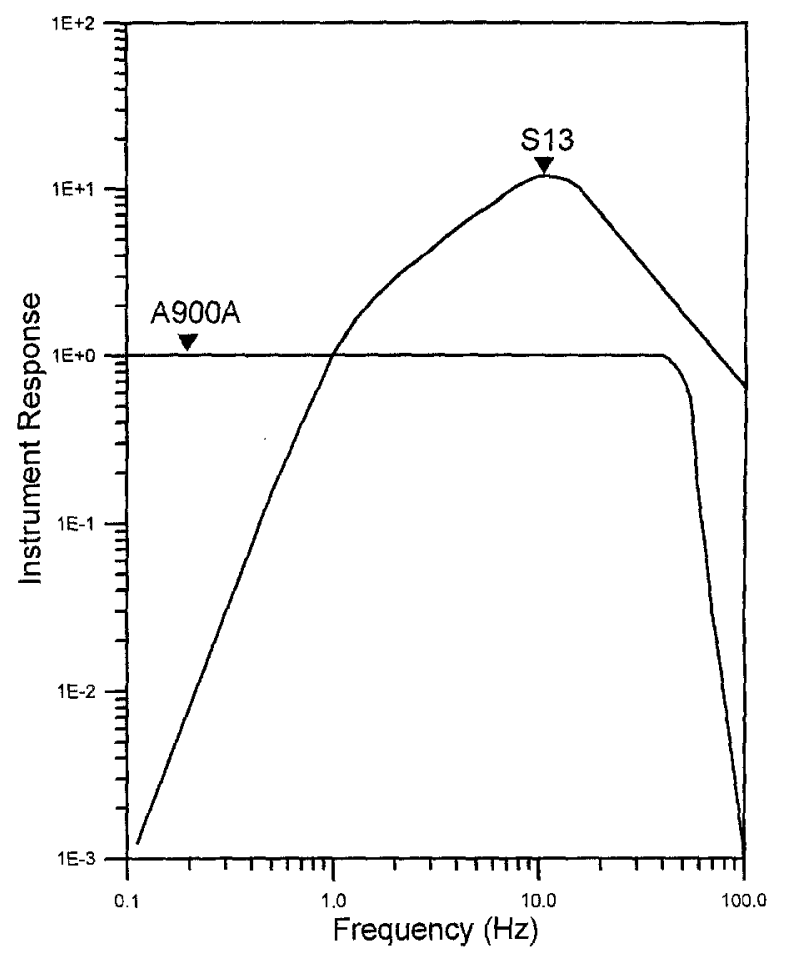

Figure 3. The system response curve of the CWBSN seismic network. The velocity sensors $(S-13)$ are operated at a peak response of 10 to $100 \mathrm{k}$ depending on the local noise conditions. The FBAs (A900A) are set at $2 \mathrm{~g}$ maximum.

sequential earthquake" at an epicentral distance much more than $100 \mathrm{~km}$ due to attenuation and geometrical spreading. In the work of both rapid reporting and early warning, the ability to reliably determine earthquake location and magnitude within a time frame of tens of seconds (a time span shorter than the time required to complete a large source rupture process) has posed a difficult problem. Other methods, which are in parallel development with the method of effective magnitude reported here, have been devised and numerically tested to determine the magnitude within the first $10 \mathrm{sec}$ after the $P$ arrivals by (1) using data from maximum displacement (fault slip), velocity, and acceleration, and to calculate a total energy sum (Teng et al., 1994; Qu, 1996), and (2) a method of recursive moment-tensor inversion (Qu and Teng, 1994). Some degree of success has been achieved, but the results are not definitive enough to be reported here. In the meantime, the real-time strong-motion monitoring of the CWBSN stations has opened up a new opportunity that provides a practical solution to this problem. The CWBSN has achieved rapid reporting well within $1 \mathrm{~min}$ after the event occurrence; that is, in $1 \mathrm{~min}$ after the first $P$-wave arrival time at the nearest station, the intensity map for a large event can be obtained, and the center of the isoseismal contours define an effective epicenter. Moreover, from the area enclosed by the 100-gal isoseismal contour curve, an empirical relationship is found that immediately gives an effective magnitude for the event. 


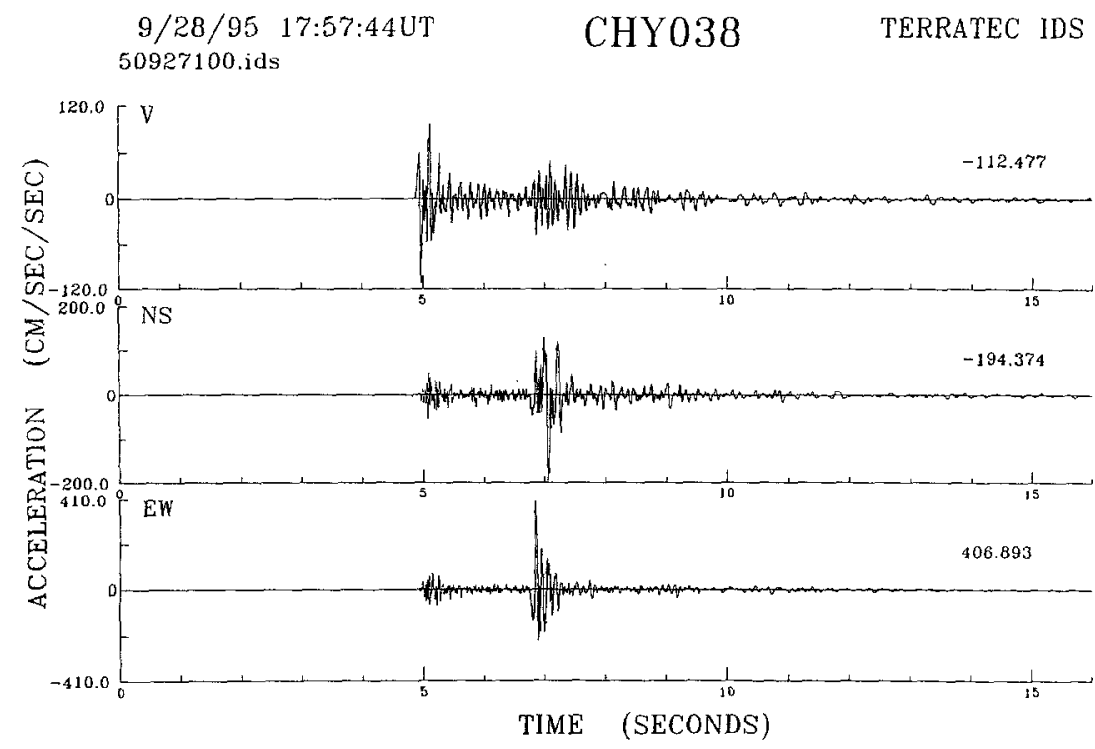

Figure 4. A sample three-component accelerogram of the CWBSN.
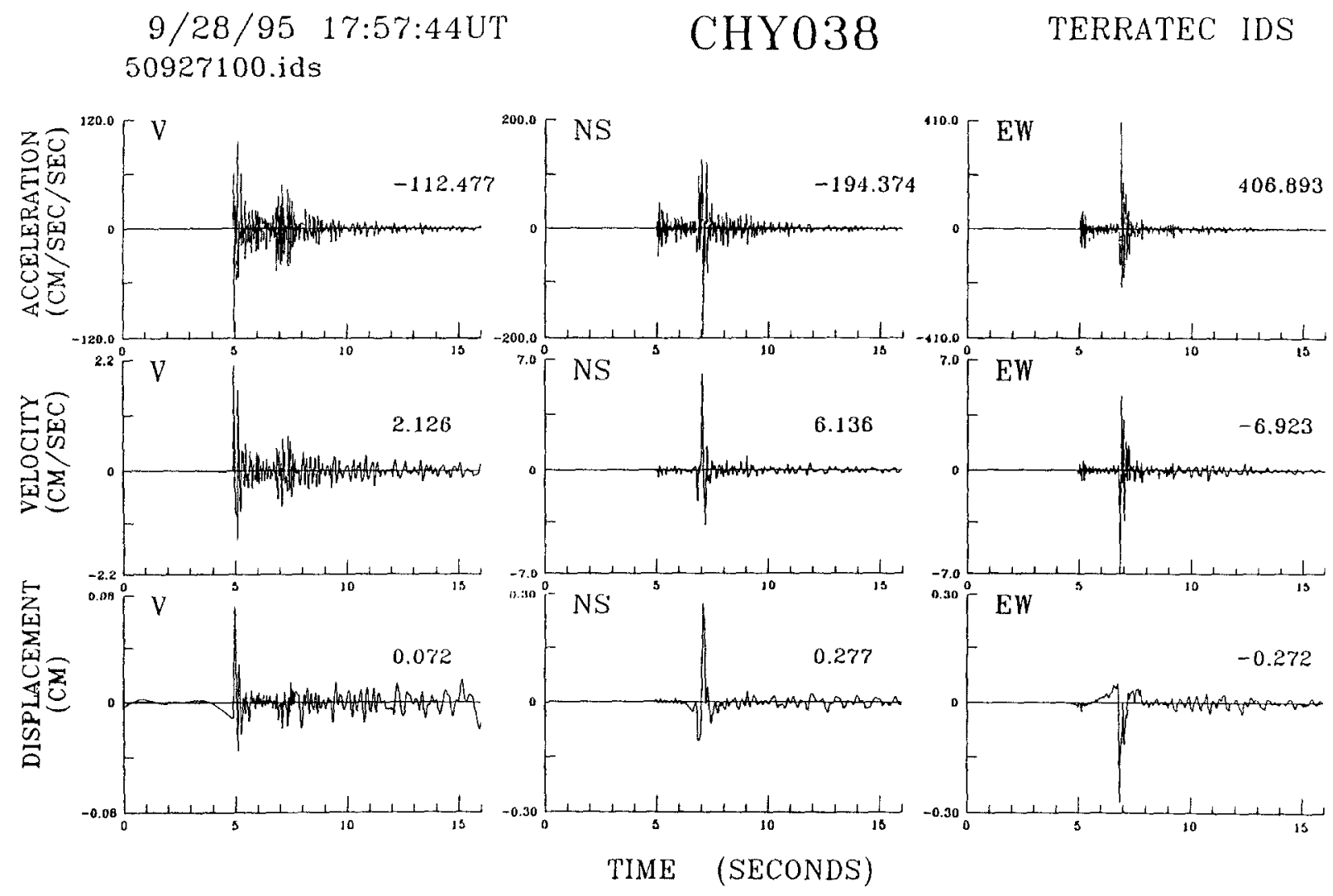

Figure 5. Velocity and displacement data from recursive integration.

\section{Real-Time Processing, Results and Discussions}

With sufficient station density, real-time strong-motion monitoring opens up an interesting new opportunity for rapid determination of the intensity map and, immediately from which, the effective epicenter and effective magnitude. We introduce the terms of effective epicenter and effective magnitude to emphasize the damage-related nature of the these quantities that are more useful in practical applications. For a large event, the effective epicenter reflects the location of maximum destructive energy release originating from a large rupture surface below. For the purpose of earthquake rapid response, the classical point-source epicenter is quite inadequate in describing where the actual damage might be. The effective magnitude reflects the degree of destructive energy. It has different emphasis than the classical magni- 


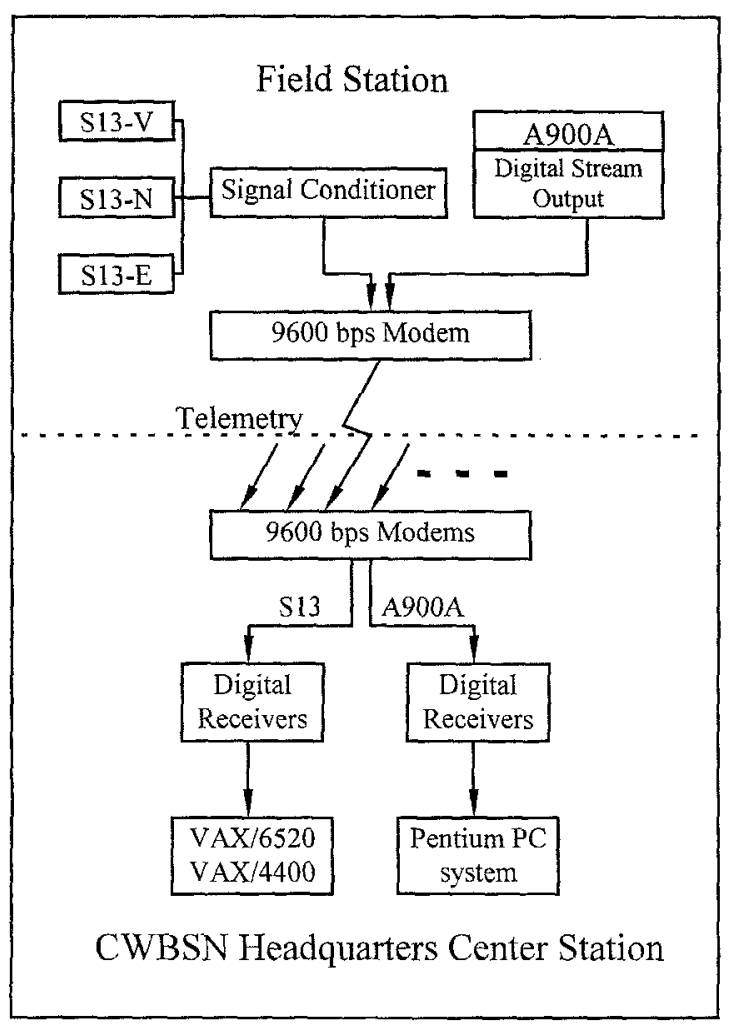

Figure 6. The digital telemetry flow chart.

tude in that the latter relates to the total energy release that may not directly reflect the degree of destruction. In either case, the focal depth is not emphasized here. For a large energy release, a deep earthquake will have a much reduced impact on the society in terms of destructive energy. It will then give a reduced effective magnitude, even though farfield recordings may still yield a large magnitude in the classical sense.

Here, we give an actual example showing how rapid reporting works in a real-time monitoring strong-motion network. Figure 7 gives the strong-motion seismogram map of an $M_{L} 6.5$ event in northeastern Taiwan, as recorded by the telemetered strong-motion network stations (solid squares). The open circle is the effective epicenter obtained in nearly real time from the strong-motion map (to be discussed later), and the solid star is the epicenter obtained (much later) by the classical least-squares hypocenter location process. The time scale and amplitude scale are shown at the lower left corner of the figure. As waves travel outward, the amplitude drops off pretty much following the regional attenuation curves. In our case, the site effects are not too important, as most of the host stations (CWBSN high-gain short-period stations) are on rock sites. A station (ENT) near the epicenter records a PGA of nearly 500 gal, but the strong motions attenuate pretty rapidly and drops to below 50 gal toward the northwest (near Taipei or the station TAP) and to below 10 gal toward the southwest (near Tainan or the station TAI). This shows that in Taiwan, which is in a tectonic region of

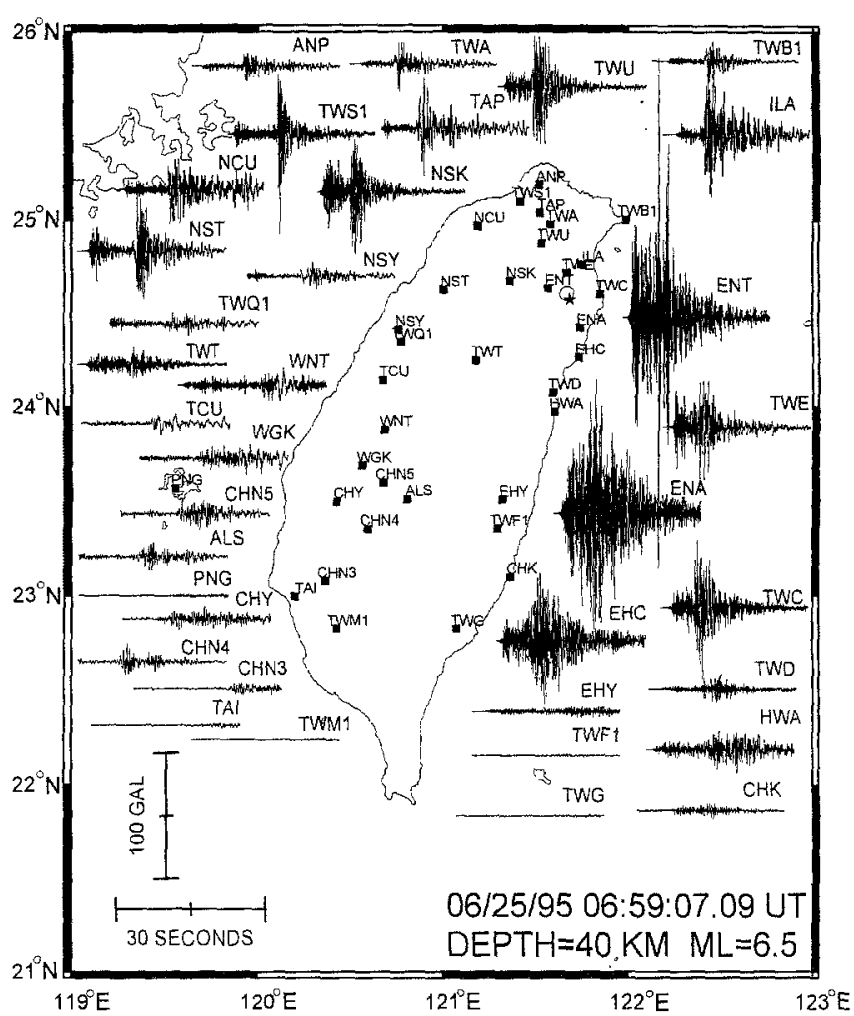

Figure 7. A strong-motion seismogram map.

modern orogeny (such as in California), the crust has a low $Q$ with strong wave attenuation. Thus an event in eastern Taiwan of magnitude less than $M 7$ may not be consequential to warrant early warning insofar as its impact to the populous western Taiwan is concerned. Effective early warning requires sufficient propagation delay time, during which the strong-motion amplitude may have already dropped with propagation distance to a level that is no longer damaging in the early warning target area. Conventional short-period seismic networks, or even broadband stations (such as the GSN/IRIS stations), will give severely clipped waveforms for near-field recording of an $M 7$ event. Well-calibrated telemetered strong-motion networks then become very useful (and perhaps the only) tool indeed for either rapid reporting or early warning work.

Strong-Motion Map. Figure 8 gives an example of what is actually happening as the multi-channel data streams of this large $M_{L} 6.5$ event are coming in under real-time strongmotion monitoring. The panel on the left of Figure 8 shows the approximate wave fronts of $S$ waves at different times after the origin time. Within about $30 \mathrm{sec}$, the peak ground accelerations (PGAs) of nearly all stations within $100 \mathrm{~km}$ of the source will have arrived in the monitoring center-in this case, the CWBSN headquarters. We shall concentrate on this region within about $100 \mathrm{~km}$ from the epicenter, because the most important and practical piece of information of the earthquake will be derived from the observations in this re- 

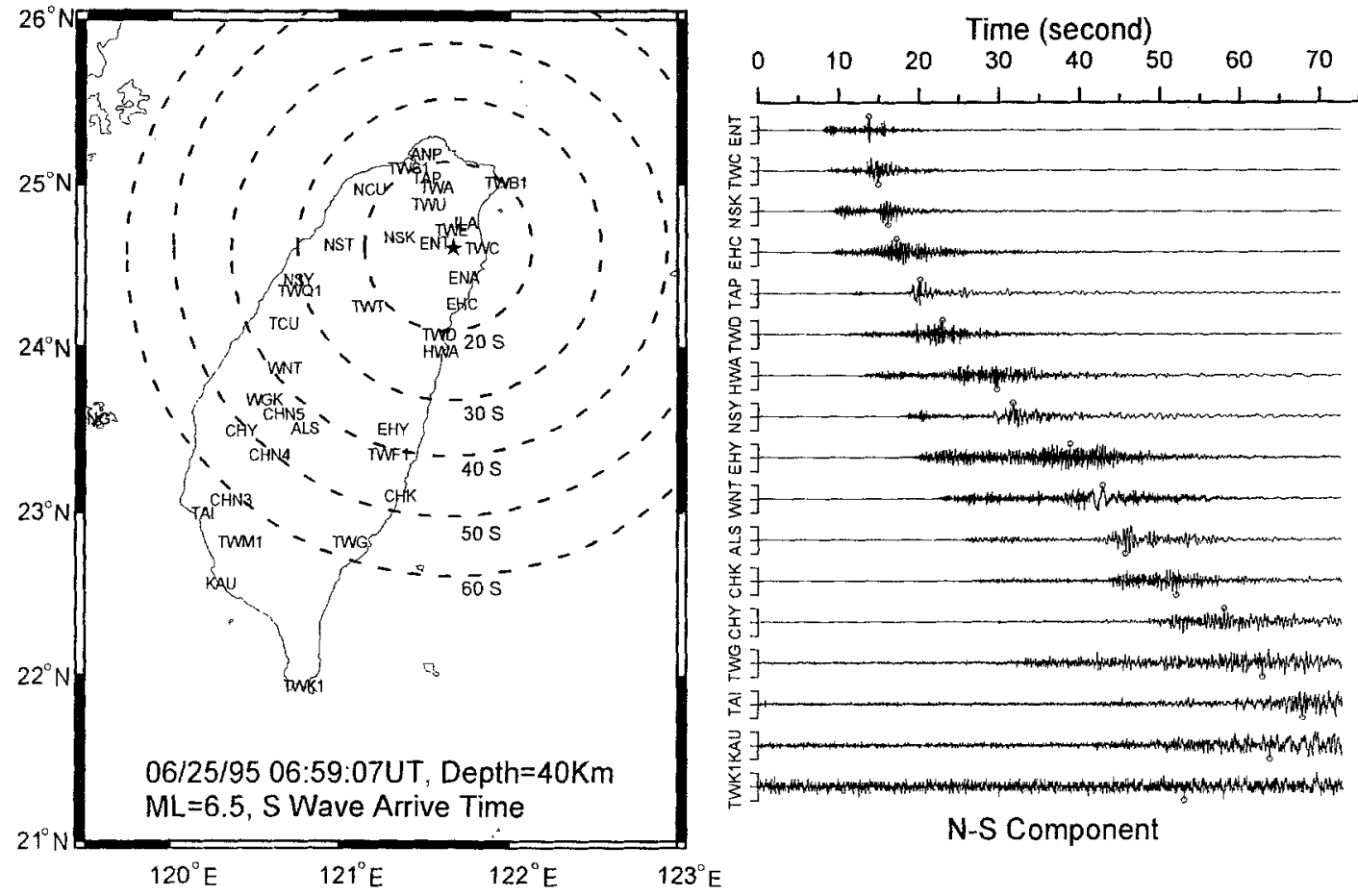

Figure 8. An event map showing the approximate 10-sec wave fronts.

gion. The panel on the right of Figure 8 shows the multichannel accelerograms. Only the $\mathrm{N}-\mathrm{S}$ components are shown in this figure; actually, all three-component data (of all $3 \times 45$ channels) are being recorded, processed, and shown up on the real-time monitor at the headquarters. The computer has already arranged the arriving signals according to their times of event triggering. The amplitudes are normalized to the PGA of each channel. Note that as the data stream of a strong-motion signal is coming into the computer, its PGA is tracked and updated until the PGA value reaches the maximum for that trace. The computer will take that value and place a small circle on that strong-motion trace to indicate where the PGA value is taken. Thus all strong-motion data streams are processed and PGAs are picked as shown in the panel on the right of Figure 8. With the real-time PGA values, the computer proceeds to construct an intensity map in a specified time interval, which in our case is every $5 \mathrm{sec}$. Figure 9 shows a sequence of intensity maps obtained as the multi-channel digital strong-motion data streams are coming in. Panel A shows the intensity map at $10 \mathrm{sec}$ after the first $P$-wave trigger time. It is rather crude, with the strongest shaking contour being only 25 gal-indicating that the strong motions represented by the principal part of the $S$-wave group have not arrived yet. At $20 \mathrm{sec}$ (panel B of Fig. 9), the contour of the strongest shaking ( 80 $\mathrm{gal}$ ) is better defined while contours in areas farther away from the source region have not yet been stabilized (again, because the principal strong motions have not completely arrived in those areas). Panels C, D, E, and F of Figure 9 show the progressive refinements of the intensity maps at, respectively, 30, 40, 50, and $60 \mathrm{sec}$ after the first $P$-wave trigger. At $60 \mathrm{sec}$, the intensity map is practically identical to the finalized version as shown in Figure 10. So, with realtime strong-motion monitoring, the intensity map is a function of time. It is important to focus our attention on the two intensity maps obtained at 20 and $30 \mathrm{sec}$. The largest and most important isoseismal contour curve (for 80 gal) has become well defined between 20 and $30 \mathrm{sec}$. It is this largest strong-motion acceleration contour curve that carries the crucial information for the effective epicenter and effective magnitude needed for rapid reporting and early warning.

Effective Epicenter. We recall that any consequential earthquake of interest to rapid reporting and early warning will have magnitude larger than $M 6$, which will have a source dimension on the order of about $10 \mathrm{~km}$. We know that the rupture initiation point-usually indicated by the classical point-source hypocenter and obtained by a least-squares inversion process, is generally not the earthquake location of practical interest. Of more practical value in terms of emergency response to a damaging earthquake would be the effective epicenter that is defined to be the center of this highest strong-motion acceleration contour curve. This definition essentially places the effective epicenter right above the most energetic ruptured asperity in a fault plane below the surface. So, we argue that, between 20 and $30 \mathrm{sec}$ after the first $P$-wave trigger, the effective epicenter is immediately known, which is located about the center of the 80-gal contour curve (in the example of Fig. 9). 

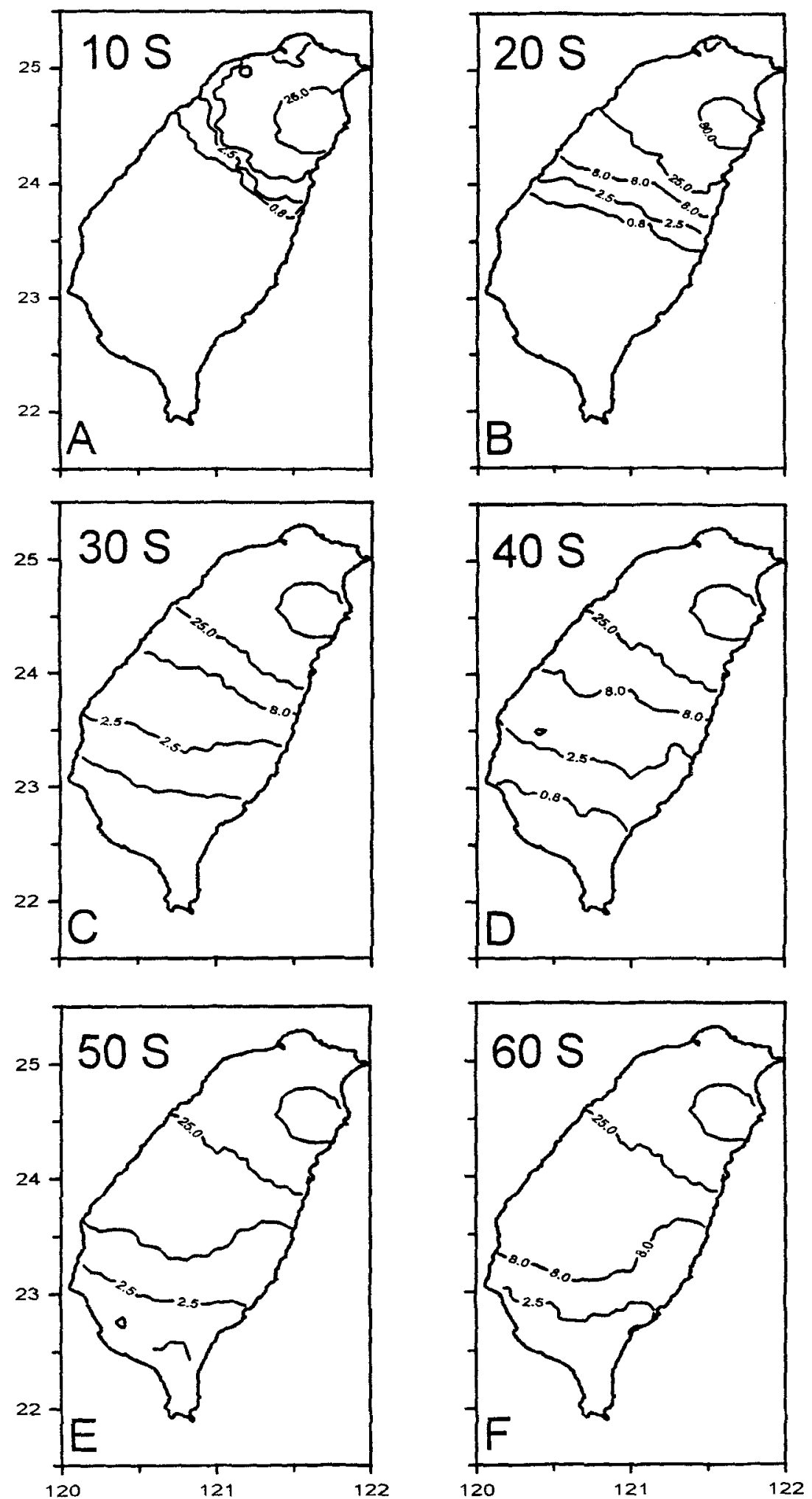

Figure 9. The strong-motion map as a function of time. Accelerations are PGA in gallons.

Effective Magnitude. The concept of the effective magnitude, in the sense of rapid reporting and early warning applications, emphasizes the degree of total surface disturbance of strong shaking produced by a large earthquake. In areas of high seismic risk, this strong surface disturbance, of course, leads to high earthquake damage. With real-time strong-motion monitoring, we have shown above that the strong-motion map can be generated within 1 min of a large event occurrence, and a straightforward method can be devised to get the effective magnitude immediately from this map. In fact, the contour of a certain large PGA value, say $100 \mathrm{gal}$, is found to be particularly useful. A 100-gal PGA 


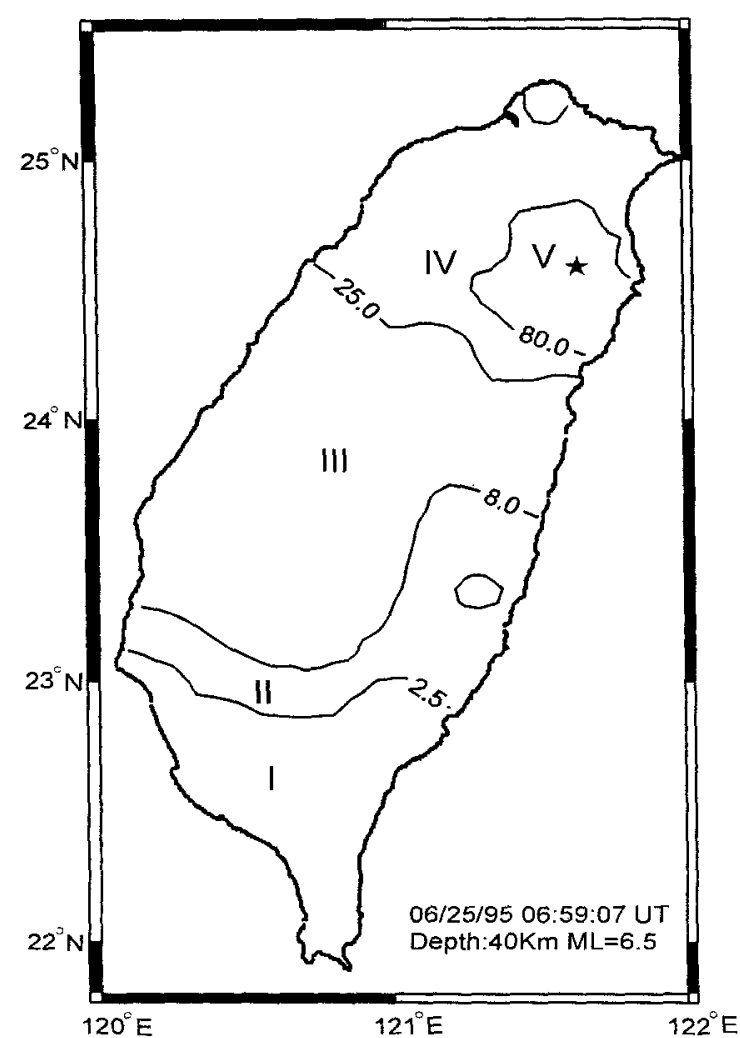

Figure 10. The final strong-motion map. Accelerations are PGA in gallons. The star indicates the location obtained later by a least-squares location routine.

corresponds to the intensity VII of the modified MM scale, and inside a zone of intensity VII, structural damage is becoming widespread. An empirical method for the determination of an effective magnitude is here introduced that is illustrated in Figure 11. Based on the acceleration data derived from well-known published intensity maps and their corresponding magnitudes of historical shallow damaging earthquakes, we have found a simple empirical relationship between the source areas covered by the 100-gal contour curve and the corresponding magnitudes of large historical earthquakes. For events larger than $M 5.5$, it is clear in Figure 11 that the logarithm of the 100 -gal source area is linearly proportional to the magnitude as shown in a semi-log plot. With this simple relationship, effective magnitude is immediately obtained as soon as the area inside the 100-gal contour curve is computed. In our example, the 80-gal PGA contour curve in Figure 10 is well defined at times between 20 and $30 \mathrm{sec}$ of the first $P$-wave arrival as explained in Figure 9. A simple routine is used to determine the area inside the 100-gal contour curve, then the linear relationship shown in Figure 11 is used to determine the effective magnitude. In this example, the derived effective magnitude is approximately $M 6.4$, a value close to the magnitude $M_{L} 6.5$ published later using moment-tensor inversion. We note that, for the rapid reporting and early warning applications,

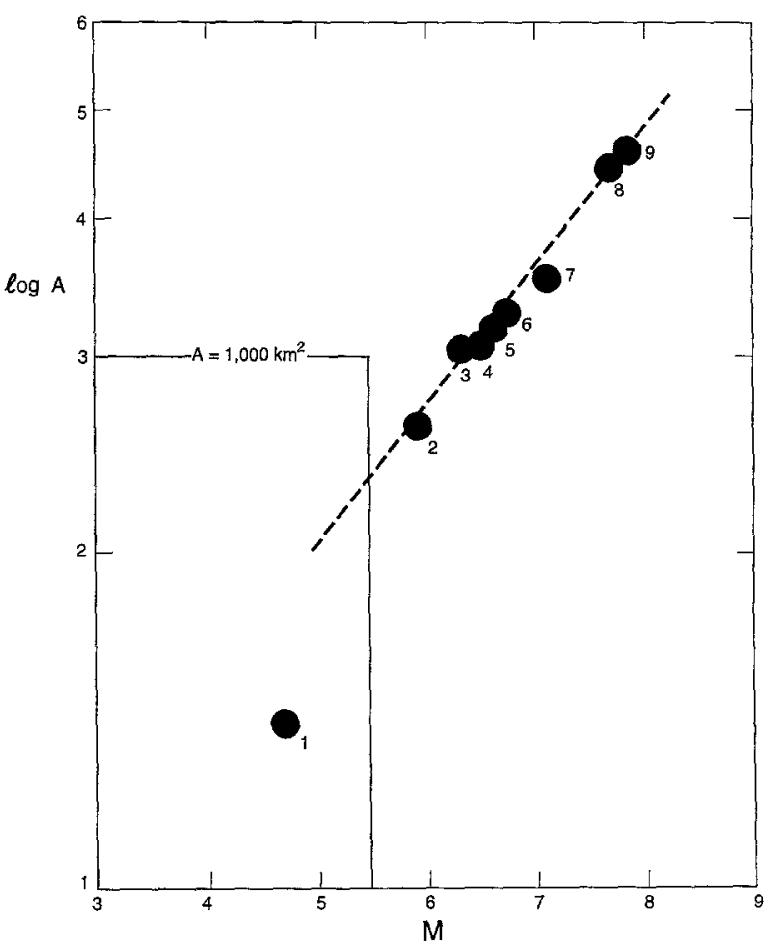

Figure 11. An empirical relationship between the area $\left(A\right.$, in $\left.\mathrm{km}^{2}\right)$ covered by the 100 -gal contour and earthquake magnitude is given by $\log y=0.114+$ $0.124 M$, where $y=\log A$. Data are compiled from the following: (1) Whittier earthquake (M 4.7), 1929 (Richter, 1958); (2) Whittier Narrow earthquake ( $M$ 5.9), 1987 (Kawase and Aki, 1990); (3) Long Beach earthquake (M 6.3), 1933 (Richter, 1958); (4) Imperial Valley earthquake (M 6.5), 1979 (Reagor et al., 1979); (5) San Fernando earthquake ( $M$ 6.6), 1971 (Scott, 1971); (6) Northridge earthquake (M 6.7), 1994 (Chang et al., 1996); (7) Loma Prieta earthquake ( $M$ 7.1), 1989 (Bolt, 1993); (8) Kern County earthquake (M 7.7), 1952 (Richter, 1958); (9) Tangshan earthquake (M 7.8), 1976 (State Seismological Bureau, 1982).

it is adequate that magnitude is determined to within about \pm 0.2 . Again, the effect of source depth has also been folded into the definition of the effective magnitude. For events of the same classical magnitude, say an $M 7$, a shallow source at depth of $20 \mathrm{~km}$ will inflict considerable heavier damage than a deeper one at $100 \mathrm{~km}$. In this sense, an $M 7$ with source depth of $100 \mathrm{~km}$ will have a considerably smaller effective magnitude as its shaking impact is considerably smaller. Here, we may note that a source of greater depth will inflict more moderate damage over a wider region due to less attenuation.

There should be no confusion between the classical definitions of epicenter and magnitude versus the "effective" definitions introduced here. The effective epicenter and effective magnitude are only used when rapid reporting and early warning are in progress. After the earthquake rapid response period, these "effective" values will be replaced 

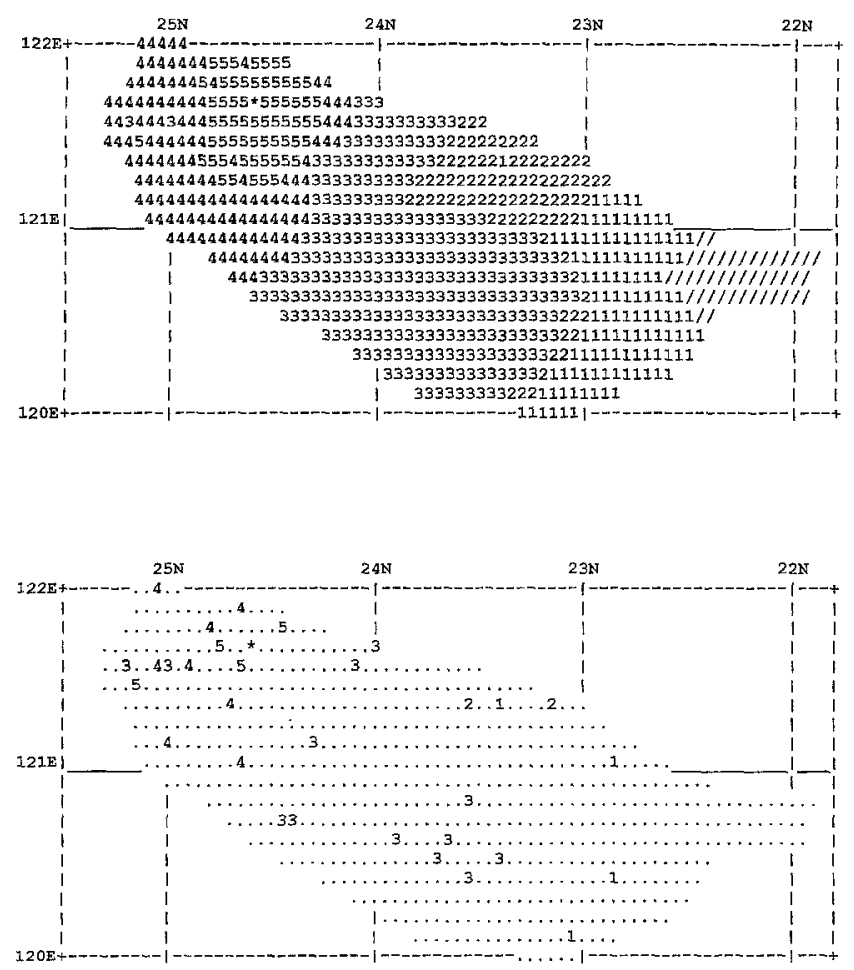

Figure 12. A Sample output through the E-mail; where the strong-motion map is plotted by letters, the size of the letter can be translated into area. An effective magnitude is obtained by a direct application of the empirical relationship in Figure 11. Numbers refer to PGA as follows: $5,>80$ gal; $4,>25$ gal; $3,>8$ gal; $2,>2.5$ gal; $1,>0.8$ gal.

with their seismological counterparts as they become available.

Finally, there is a PC in the CWBSN system that is in charge of information dissemination. It organizes the findings and electronically transmits the information to subscribing users, who, from that point on, will execute whatever actions that are appropriate with the given rapid reporting or early warning information. Figure 12 is a sample of the strong-motion map part of the electronic message transmitted via the E-mail in a test case. This test run has been successfully conducted for the past 3 months, the E-mail message carries nearly real time the rapid reporting message from Taiwan across the Pacific to the authors in California whenever an $M>5$ event occurs. In realistic situations when the CWBSN rapid reporting and early warning system is approved by the authorities for routine operation, the subscribing users, such as the county and city offices of emergency services, the police departments, nuclear power plants, and any earthquake-safety-sensitive governmental and private agencies, will have to "hard-wire" their accesses to the CWBSN computer with special communication channels that will minimize or eliminate possible communication delays or breakdowns.

\section{Acknowledgments}

We thank Professor Ching Yen Tsay, Director of Taiwan's Central Weather Bureau, whose leadership is instrumental in much of the recent progress in observational seismology in Taiwan. Thanks are due to Yeong Tein Yeh and George Liu, in the Institute of Earth Sciences, Academia Sinica; and Yu Lung Liu, Kuen Sung Liu, and many of their colleagues in the Observation Seismology Center of the Taiwan's Central Weather Bureau; without their dedication, the real-time capability of the CWBSN could not deliver the excellent data as illustrated in this article. We also thank Hiroo Kanamori and Egill Hauksson of Caltech Seismological Laboratory and Y. Nakamura of Japan Railways for many stimulating discussions, and the reviewers and Kenneth W. Campell who have made valuable suggestions in the presentation of this article. This research is funded by a Central Weather Bureau, Republic of China grant on Earthquake Early Warning and Strong-Motion Instrumentation Program and by the U.S. Geological Survey Contract Number 14-08-0001-A0260.

\section{References}

Bakun, B. (1990). Early warning alert esteem, U.S. Geological Survey, Menlo Park, Public Affairs Memo.

Bolt, B. (1993). Earthquakes, W. H. Freeman and Co., New York, p. 261.

Chang, S. W., J. D. Bray, and R. B. Seed (1996). Engineering implications of ground motions from the Northridge earthquake, Bull. Seism. Soc. Am. 86, S270-S288.

Espinosa Aranda, J. M., A. Jimenez, G. Ibarrola, F. Alcantar, A. Aguilar, M. Inostroza, and S. Maldonado (1995). Mexico City seismic alert system, Seism. Res. Lett. 66, $42-53$.

Heaton, T. H. (1985). A model for seismic computerized alert network, Science 228, 987-990.

Holden, R., R. Lee, and M. Reichle (1989). Technical and Economic Feasibility of an Earthquake Warning System in California, Calif. Div. Mines Geol. Spec. Publ. 101.

Kawase, H. and K. Aki (1990). Topography effect and critical SV-wave incidence: possible explanation of damage pattern by the Whittier Narrows, California, earthquake of 1 October 1987, Bull. Seism. Soc. Am. 80, 1-22.

Lee, W. H. K. (1993). A project implementation plan for an advanced earthquake monitoring system. Research Report of Central Weather Bureau, Taipei, Taiwan, R.O.C., No. 444, also as U.S. Geol. Surv. Open-File Rept. 94-004, 89 pp.

Lee, W. H. K. and S. T. Houck (1977). Identifying high-seismicity regions of the world for earthquake-prediction studies, U.S. Geol. Surv. OpenFile Rept. 77-693, 134 pp.

Lee, W. H. K., T. C. Shin, and T. L. Teng (1996). Design and implementation of earthquake early warning system in Taiwan. Paper No. 2133, 11th World Conference of Earthquake Engineering, Acapulco, Mexico.

Nakamura, Y. (1989). Earthquake alarm system for Japan railways, Japanese Railway Eng. 28, no. 4, 3-7.

National Research Council of the National Academy of Science. (1991). Real-time earthquake monitoring, Report from the Committee on Seismology, National Academy Press, Washington, D.C.

Reagor, B. G., C. W. Stover, S. T. Algermissen, K. V. Steinbrugge, P. Hubiak, M. G. Hopper, and L. M. Barnhard (1979). Preliminary evaluation of the distribution of seismic intensities, U.S. Geol. Surv. Profess. Pap. 1254, 251-258.

Richter, C. F. (1958). Elementary Seismology, W. H. Freeman and Co,, New York, pp. 39 and 519.

Qu, J. (1996). Part II: Recursive stochastic deconvolution in the estimation of earthquake source parameters, Ph.D. Thesis, University of Southern California.

Qu, J. and T. L. Teng (1994). Recursive stochastic deconvolution in the estimation of earthquake source parameters: synthetic waveforms, Phys. Earth Planet. Interiors 86, 301-327. 
Scott, N. H. (1971). Preliminary report on felt area and intensity, U.S. Geol. Surv. Profess. Pap. 773, 153-154.

Shin, T. C. (1993). Progress summary of the Taiwan Strong Motion Instrumentation Program, Symposium Taiwan Strong Motion Instrumentation Program, 1-10 (in Chinese).

Shin, T. C., Y. B. Tsai, and Y. M. Wu (1996). Rapid response of large earthquakes in Taiwan using a real-time telemetered network of digital accelerographs, Paper No. 2137, 11th World Conference of Earthquake Engineering, Acapulco, Mexico.

State Seismological Bureau "1976 Tangshan Earthquake" Editor Group (1982). 1976 Tangshan Earthquake, Earthquake Publication Service, p. 4.

Teng, T.-l., M. Hsu, W. H. K. Lee, Y. B. Tsai, F. T. Wu, Y. T. Yeh, and G. Liu (1994). Annual Report to the Central Weather Bureau on Earthquake Early Warning and Implementation of the Strong Motion Instrumentation Program.

Teng, T.-1., M. Hsu, W. H. K. Lee, Y. B. Tsai, F. T. Wu, and G. Liu (1996). Annual Report to the Central Weather Bureau on Earthquake Early Warning and Implementation of the Strong Motion Instrumentation Program.

Toksoz, M. N., A. M. Dainty, and J. T. Bullit (1990). A prototype earthquake warning system for strike-slip earthquakes, Pure Appl. Geophys. 133, no. 3, 475-487.
Wald, L. A., L. K. Hutton, J. Mori, D. G. Given, and L. M. Jones (1991). The southern California Network Bulletin, January-December, 1990. Wu, Y. M. (1996). E-mail message to W. H. K. Lee, 6 May, 1996.

Department of Earth Sciences

University of Southern California

Los Angeles, California

(T.-I.T., W.H.K.L.)

Central Weather Bureau

Taipei, Taiwan, ROC

(L.W., T.-C.S.)

Institute of Geophysics

National Central University

Chungli, Taiwan ROC

(Y.-B.T.)

Manuscript received 13 August 1996. 